\title{
660 TARGETING T CELL FATES: CONVERTING EXHAUSTION TO MEMORY TO IMPROVE IMMUNOTHERAPEUTIC RESPONSES TO CANCER
}

Nicole Scharping*, Allison Cafferata, Maximilian Heeg, Quynhanh Nguyen, Ananda Goldrath. University of California San Diego, La Jolla, CA, United States

Background In cancer, CD8 $+\mathrm{T}$ cells have the power to target and kill tumor cells with precision, but often fail due to chronic activation from the immunosuppressive tumor microenvironment (TME). T cells that experience prolonged activation in the TME differentiate into a severely dysfunctional cell state known as exhaustion. In healthy tissues, $\mathrm{T}$ cells differentiate into tissue-resident memory cells (TRM) in response to infection, which remain lodged in tissues to provide protection from reinfection. When TRM-like cells are found in patient tumors, they are correlated with improved outcomes and responses to immunotherapy. Understanding how to manipulate $\mathrm{T}$ cell fates in an effort to prevent exhaustion and favor TRM-characteristics could benefit cancer immunotherapy.

Methods To explore differences between these cell states, we screened the core TRM gene-expression signatures for genes downregulated as $\mathrm{T}$ cells undergo terminal exhaustion. Targets were then overexpressed in antigen-specific $\mathrm{T}$ cells and adoptively transferred into tumor-bearing mice for analysis.

Results Interestingly, many genes related to protein regulation and processing were identified, including a novel gene called Neuralized E3 Ubiquitin Protein Ligase 3 (Neurl3). Neurl3's function is not well described, however, experimentally mutating the RING domain suggests Neurl3 transfers ubiquitin to target proteins for degradation. When Neurl3 was overexpressed in tumor-specific $\mathrm{T}$ cells, we found tumor infiltrating lymphocytes still upregulated inhibitory receptors PD1 and Tim3, but showed enhanced anti-tumor function. Neurl3-overexpressing $\mathrm{T}$ cells had increased accumulation in the TME, upregulated canonical TRM markers CD69 and CD103, produced more cytokines, controlled tumor growth, and increased mouse survival in B16 melanoma.

Conclusions These results highlight the understudied field of negative regulation of $\mathrm{T}$ cell function by protein degradation in $\mathrm{T}$ cell differentiation fate and uncover a potential gene target for immunocellular therapies to favor functional $\mathrm{T}$ cell fates in cancer.

Ethics Approval The study was approved by UCSD's Institutional Animal Care and Use Committee, protocol number S04105.

http://dx.doi.org/10.1136/jitc-2021-SITC2021.660 